\title{
FACTORIZATION OF UNIFORMLY CONTINUOUS MAPS THROUGH UNIFORM SHAPE FIBRATIONS
}

\author{
TAKAHISA MiYATA \\ Kobe University, Japan
}

\begin{abstract}
It is well-known that every continuous map is the composite of a homotopy equivalence and a fibration. In this paper, we introduce the notion of uniform shape fibration, and show that every uniformly continuous map is the composite of a uniform shape equivalence and a uniform shape fibration.
\end{abstract}

\section{INTRODUCTION}

In algebraic topology one often replaces continuous maps by fibrations, using the well-known fact that every continuous map $f: X \rightarrow Y$ between spaces is the composite of a homotopy equivalence and a fibration. However, the homotopy lifting property is not a suitable property for continuous maps between spaces with bad local properties. To overcome the deficiency, S. Mardešić and T. B. Rushing introduced in [5] the notion of shape fibration between compact metric spaces, extending the notion of the approximate homotopy lifting property introduced by D. S. Coram and P. F. Duvall ([1], see also [4] for the definition of shape fibration between arbitrary topological spaces).

It is natural to raise the question: is every continuous map between spaces the composite of a shape equivalence and a shape fibration? However, when one tries to solve the problem, one easily faces a difficulty in handling product spaces of the form $Y \times C(I, X)$ where $C(I, X)$ is the space of continuous maps from $I=[0,1]$ to $X$ with the compact-open topology, since the function space is almost never compact. In fact, the factorization of $f: X \rightarrow Y$ is given by a homotopy equivalence $h: X \rightarrow E_{f}$ and a fibration $g: E_{f} \rightarrow Y$, where

2010 Mathematics Subject Classification. 55P55, 54E15.

Key words and phrases. Uniform shape fibration, uniform homotopy lifting property, factorization axiom. 
$E_{f}=\{(x, \omega) \in X \times C(I, Y): f(x)=\omega(0)\}$, and $h$ and $g$ are defined by $h(x)=\left(x, e_{f(x)}\right)$ and $g(x, \omega)=\omega(1)$, respectively. Here, for each $y \in Y$, $e_{y}: I \rightarrow Y$ is the path defined by $e_{y}(t)=y$ for all $t \in I$.

In this paper, to overcome this difficulty, we consider this problem in the framework of uniform metric spaces. We introduce the notion of uniform shape fibration for uniformly continuous maps and prove that every uniformly continuous map between metric spaces is the composite of a uniform shape equivalence (in the sense of [7]) and a uniform shape fibration.

For any category $\mathcal{C}$, let inv- $\mathcal{C}$ denote the category of inverse systems in $\mathcal{C}$. If $\boldsymbol{X}=\left(X_{i}, p_{i, i+1}\right)$ is a tower, let $p_{i j}=p_{i, i+1} \circ p_{i+1, i+2} \circ \cdots \circ p_{j-1, j}$ for $i<j$ and $p_{i i}=1_{X_{i}}$, the identity morphism on $X_{i}$. For any subcategory $\mathcal{D}$ of $\mathcal{C}$, a tower $\boldsymbol{X}=\left(X_{i}, p_{i, i+1}\right)$ in inv- $\mathcal{C}$ is called a $\mathcal{D}$-tower if all $X_{i}$ are objects of $\mathcal{D}$ and all $p_{i, i+1}$ are morphisms of $\mathcal{D}$. A level morphism $\left(f_{i}\right): \boldsymbol{X} \rightarrow \boldsymbol{Y}$ between towers $\boldsymbol{X}=\left(X_{i}, p_{i, i+1}\right)$ and $\boldsymbol{Y}=\left(Y_{i}, q_{i, i+1}\right)$ consists of morphisms $f_{i}: X_{i} \rightarrow Y_{i}$ such that $f_{i} \circ p_{i, i+1}=q_{i, i+1} \circ f_{i+1}$. Let pro- $\mathcal{C}$ denote the procategory of $\mathcal{C}$. For more details on inv- $\mathcal{C}$ and pro- $\mathcal{C}$, the reader is referred to $[6$, Chapter 1, §1.1].

In this paper, we consider the category UM of metric spaces and uniformly continuous maps. Unless otherwise stated, metric spaces are assumed to possess the uniformities induced by their metrics.

Here is our main result.

THEOREM 1.1. Every uniformly continuous map $f: X \rightarrow Y$ between metric spaces admits a metric space $E$ and uniformly continuous maps $h$ : $X \rightarrow E$ and $g: E \rightarrow Y$ with the following properties:

(1) $f=g \circ h$,

(2) $h$ induces a uniform shape equivalence, and

(3) $g$ is a uniform shape fibration.

\section{UNIFORM ABSOLUTE NEIGHBORHOOD RETRACTS AND UNIFORM RESOLUTIONS}

For any $\varepsilon>0$, two points $y, y^{\prime}$ of a metric space $Y$ are $\varepsilon$-near provided $d\left(y, y^{\prime}\right)<\varepsilon$, and any two uniformly continuous maps $f, g: X \rightarrow Y$ of a topological space $X$ into a metric space $Y$ are $\varepsilon$-near provided $\rho(f, g)=$ $\sup \{d(f(x), g(x)): x \in X\}<\varepsilon$.

A metric space $Y$ is a uniform absolute neighborhood retract (uniform $A N R$, in short) (resp., uniform absolute retract (uniform $A R$, in short)) if, whenever $Y$ is uniformly embedded in a metric space $X$, there exist a uniform neighborhood $U$ of $Y$ and a uniform retraction $r: U \rightarrow Y$ (resp., there exists a uniform retraction $r: X \rightarrow Y$ ). This is equivalent to the condition that whenever $A$ is a subset of a metric space and a uniformly continuous map $f: A \rightarrow Y$, there exist a uniform neighborhood $U$ of $A$ and a uniformly continuous map $\bar{f}: U \rightarrow Y$ (resp., $\bar{f}: X \rightarrow Y$ ) which extends $f$. Every metric 
space that is a uniform ANR (resp., uniform AR) in its metric uniformity is an ANRU (resp., ARU) in the sense of Isbell's book [3].

For any metric spaces $\left(X, d_{X}\right)$ and $\left(Y, d_{Y}\right)$, let the Cartesian product $X \times$ $Y$ have the metric $d$ defined by $d\left((x, y),\left(x^{\prime}, y^{\prime}\right)\right)=\max \left\{d_{X}\left(x, x^{\prime}\right), d_{Y}\left(y, y^{\prime}\right)\right\}$. Then $X \times Y$ is a product in the category UM. We call $X \times Y$ with the metric uniformity the uniform product of $X$ and $Y$ in order to distinguish this from another type called semi-uniform product, which will be explained in the following section.

The following properties will be used later in this paper. Their proofs are similar to that for ANR's (see Propositions 4.1, 6.1, and Theorems 3.1, 3.2 of $[2])$.

Proposition 2.1. (1) If $X$ and $Y$ are uniform $A N R$ 's, then so is $X \times Y$.

(2) If $X$ is a uniform ANR, then every uniform neighborhood in $X$ is a uniform ANR.

Let UANR denote the full subcategory of UM whose objects are uniform ANR's. Let $\mathbb{N}$ denote the set of positive integers.

A uniform UANR-resolution $\boldsymbol{p}: X \rightarrow \boldsymbol{X}$ of a metric space $X$ consists of a UANR-tower $\boldsymbol{X}=\left(X_{i}, p_{i, i+1}\right)$ and a morphism $\boldsymbol{p}=\left(p_{i}\right): X \rightarrow \boldsymbol{X}$ in inv- UM satisfying the following two conditions:

$\left(\mathrm{R}_{1}\right)$ For every uniformly continuous map $f: X \rightarrow P$ to a uniform ANR $P$ and for every $\varepsilon>0$, there exist $i \in \mathbb{N}$ and a uniformly continuous map $h: X_{i} \rightarrow P$ such that $\rho\left(f, h \circ p_{i}\right)<\varepsilon$, and

$\left(\mathrm{R}_{2}\right)$ For every uniform ANR $P$ and for every $\varepsilon>0$, there exists $\delta>0$ such that whenever $f, f^{\prime}: X_{i} \rightarrow P$ are uniformly continuous maps with $\rho\left(f \circ p_{i}, f^{\prime} \circ p_{i}\right)<\delta$, then there exists $i^{\prime} \in \mathbb{N}$ such that $i^{\prime} \geq i$ and $\rho\left(f \circ p_{i i^{\prime}}, f^{\prime} \circ p_{i i^{\prime}}\right)<\varepsilon$.

A uniform resolution of a uniformly continuous map $f: X \rightarrow Y$ between metric spaces $X$ and $Y$ is a triple $(\boldsymbol{p}, \boldsymbol{q}, \boldsymbol{f})$ which consists of uniform UANRresolutions $\boldsymbol{p}=\left(p_{i}\right): X \rightarrow \boldsymbol{X}$ and $\boldsymbol{q}=\left(q_{i}\right): Y \rightarrow \boldsymbol{Y}$ of $X$ and $Y$, respectively, and a level morphism $\boldsymbol{f}=\left(f_{i}\right): \boldsymbol{X} \rightarrow \boldsymbol{Y}$ such that $q_{i} \circ f=f_{i} \circ p_{i}$ for each $i \in \mathbb{N}$.

For any set $S$, let $\ell^{\infty}(S)$ denote the set of all real valued bounded functions $f: S \rightarrow \mathbb{R}$ with the metric $d$ defined by $d(f, g)=\sup \{|f(x)-g(x)|: x \in S\}$.

THEOREM 2.2. (1) Every metric space admits a uniform UANR-resolution.

(2) Every uniformly continuous map between metric spaces admits a uniform resolution.

Proof. (1): Every metric space $X$ is isometrically embedded in $\ell^{\infty}(X)$, which is a uniform AR (see Proposition 14 and Theorem 15 of [3]). For each $i \in \mathbb{N}$, let $X_{i}$ be the $1 / 3^{i}$-neighborhood of $X$ in $\ell^{\infty}(X)$, and let $p_{i}: X \hookrightarrow X_{i}$ 
and $p_{i, i+1}: X_{i+1} \hookrightarrow X_{i}$ be the inclusion maps. Then a morphism $\boldsymbol{p}=\left(p_{i}\right)$ : $X \rightarrow \boldsymbol{X}=\left(X_{i}, p_{i, i+1}\right)$ is a uniform UANR-resolution of $X$. Indeed, each $X_{i}$ is a uniform ANR since it is a uniform neighborhood in a uniform AR. Condition $\left(\mathrm{R}_{1}\right)$ easily follows from the definition of uniform ANR. To verify condition $\left(\mathrm{R}_{2}\right)$, let $P$ be a uniform ANR, and let $\varepsilon>0$. Let $f, f^{\prime}: X_{i} \rightarrow P$ be uniformly continuous maps such that $\rho\left(f \circ p_{i}, f^{\prime} \circ p_{i}\right)<\varepsilon / 3$, and choose $i^{\prime} \in \mathbb{N}$ with $i^{\prime}>i$ so that if $x, x^{\prime} \in X_{i}$ and $d\left(x, x^{\prime}\right)<1 / 3^{i^{\prime}}$, then $d\left(f(x), f\left(x^{\prime}\right)\right)<\varepsilon / 3$ and $d\left(f^{\prime}(x), f^{\prime}\left(x^{\prime}\right)\right)<\varepsilon / 3$. Then $\rho\left(f \circ p_{i i^{\prime}}, f^{\prime} \circ p_{i i^{\prime}}\right)<\varepsilon$ follows. For, for each $x \in X_{i^{\prime}}, d\left(x, x^{\prime}\right)<1 / 3^{i^{\prime}}$ for some $x^{\prime} \in X$, and $d\left(f \circ p_{i i^{\prime}}(x), f^{\prime} \circ p_{i i^{\prime}}(x)\right)<d(f \circ$ $\left.p_{i i^{\prime}}(x), f \circ p_{i i^{\prime}}\left(x^{\prime}\right)\right)+d\left(f \circ p_{i i^{\prime}}\left(x^{\prime}\right), f^{\prime} \circ p_{i i^{\prime}}\left(x^{\prime}\right)\right)+d\left(f^{\prime} \circ p_{i i^{\prime}}\left(x^{\prime}\right), f^{\prime} \circ p_{i i^{\prime}}(x)\right)<\varepsilon$.

$(2)$ : Let $\boldsymbol{p}=\left(p_{i}\right): X \rightarrow \boldsymbol{X}=\left(X_{i}, p_{i, i+1}\right)$ and $\boldsymbol{q}=\left(q_{i}\right): Y \rightarrow \boldsymbol{Y}=$ $\left(Y_{i}, q_{i, i+1}\right)$ be the uniform UANR-resolutions as above, and let $f: X \rightarrow Y$ be a uniformly continuous map. Then, since $\ell^{\infty}(Y)$ is a uniform AR, there exists a uniformly continuous map $\bar{f}: \ell^{\infty}(X) \rightarrow \ell^{\infty}(Y)$ such that $\bar{f}(x)=f(x)$ for $x \in X$. We can find an increasing sequence $\left\{k_{i}: i \in \mathbb{N}\right\}$ so that the restrictions $f_{i}: X_{k_{i}} \rightarrow Y_{i}$ of $\bar{f}$ are well-defined. Let $\boldsymbol{X}^{\prime}=\left(X_{i}^{\prime}, p_{i, i+1}^{\prime}\right)$, where $X_{i}^{\prime}=X_{k_{i}}$ and $p_{i, i+1}^{\prime}=p_{k_{i} k_{i+1}}$. Then we have a level morphism $\boldsymbol{f}=\left(f_{i}\right): \boldsymbol{X}^{\prime} \rightarrow \boldsymbol{Y}$. The restriction $\boldsymbol{p}^{\prime}=\left(p_{i}^{\prime}\right): X \rightarrow \boldsymbol{X}^{\prime}$ of $\boldsymbol{p}$, where $p_{i}^{\prime}=p_{k_{i}}$, forms a uniform UANR-resolution. The morphism $\boldsymbol{f}$ together with $\boldsymbol{p}^{\prime}$ and $\boldsymbol{q}$ forms a uniform resolution of $f$ as required.

\section{UNIFORM HOMOTOPIES}

A map $f: X \times Y \rightarrow Z$ for metric spaces $X$ and $Y$ to a uniform space $Z$ is semi-uniform if the family $\{f(\cdot, y): y \in Y\}$ is equiuniformly continuous on $X$ and for each $x \in X, f(x$,$) is a uniformly continuous map on Y$. The semi-uniform product $X * Y$ is the Cartesian product $X \times Y$ with the weak uniformity induced by all the semi-uniform maps on $X \times Y$ to uniform spaces (see [3, p. 44]).

Let $U(X, Y)$ denote the set of all uniformly continuous maps $f: X \rightarrow Y$ with the metric $d$ defined by $d(f, g)=\sup \{d(f(x), g(x)): x \in X\}$.

The following properties of semi-uniform products are useful (see Propositions 23,26 of [3]).

Proposition 3.1. (1) There is a uniform equivalence $U(X, U(Y, Z)) \approx$ $U(X * Y, Z)$.

(2) The uniformity of $X * Y$ is generated by the uniform covers of the form $\left\{U_{\alpha} \times V_{\beta}^{\alpha}\right\}$, where $\left\{U_{\alpha}\right\}$ is a uniform cover of $X$ and for each $\alpha,\left\{V_{\beta}^{\alpha}\right\}$ is a uniform cover of $Y$.

The following property is also used later in this paper.

Proposition 3.2. If $Y$ is a uniform ANR, then for every metric space $X, U(X, Y)$ is a uniform $A N R$. 
Proof. The proof is similar to that for ANR's (see Theorem 4, p. 38, of [6]). Here we use the following two facts:

- Each uniformly continuous map $f: A \rightarrow U(X, Y)$, where $A$ is a subset of a metric space $Z$, corresponds to a uniformly continuous map $F$ : $A * X \rightarrow Y$ (see Proposition 3.1 (1)).

- For each uniform neighborhood $W$ of $A * X$ in $Z * X$, there exists a uniform neighorhood $U$ of $A$ in $Z$ such that $A * X \subset U * X$ (see Proposition $3.1(2))$.

Let $f, g: X \rightarrow Y$ be uniformly continuous maps between metric spaces. Then a uniform homotopy from $f$ to $g$ is a uniformly continuous map $H$ : $X * I \rightarrow Y$ such that $H(\cdot, 0)=f$ and $H(\cdot, 1)=g . f$ is uniformly homotopic to $g$ if there is a uniform homotopy from $f$ to $g$. This definition differs from that of Isbell [3, p. 82], who defines a uniform homotopy as a uniformly continuous map $H: X \times I \rightarrow Y$ on the uniform product. Since the semiuniform product is finer than the uniform product, every uniform homotopy in Isbell's definition is a uniform homotopy in our sense but not conversely. In the case that $X$ is compact, those definitions coincide. An important fact for our uniform homotopy is that there is a uniform equivalence $U(X, U(I, Y)) \approx$ $U(X * I, Y)$ (see Proposition $3.1(1)$ ).

For each $\delta>0$, a uniform homotopy $f: X * I \rightarrow Y$ is a uniform $\delta$ homotopy if for each $x \in X$, the diameter of the set $\{H(x, t): t \in I\}$ is less than $\delta$.

A uniformly continuous map $f: X \rightarrow Y$ is a uniform homotopy equivalence if there exists a uniformly continuous map $g: Y \rightarrow X$ such that $g \circ f$ and $f \circ g$ are uniformly homotopic to the identity maps on $X$ and $Y$, respectively.

Proposition 3.3. Let $Y$ be a uniform ANR. Then for each $\varepsilon>0$, there exists $\delta>0$ such that any two $\delta$-near uniformly continuous maps into $Y$ are uniformly $\varepsilon$-homotopic.

Proof. Embed $Y$ isometrically in $\ell^{\infty}(Y)$, and let $r: U \rightarrow Y$ be a uniform retraction from a uniform neighborhood $U$ of $Y$. Let $\delta>0$ be such that the $\delta$-neighborhood of $Y$ is contained in $U$, and for any $x, x^{\prime} \in U$, if $d\left(x, x^{\prime}\right)<\delta$, then $d\left(r(x), r\left(x^{\prime}\right)\right)<\varepsilon$. If $f, f^{\prime}: X \rightarrow Y$ are $\delta$-near uniformly continuous maps, then the map $H: X * I \rightarrow Y$ defined by $H(x, t)=r\left((1-t) f(x)+t f^{\prime}(x)\right)$ is a uniform $\varepsilon$-homotopy from $f$ to $f^{\prime}$.

Using this uniform homotopy, one can define the corresponding shape category as the abstract shape category for the pair of categories (HUM, HUANR), where HUM is the category of metric spaces and uniform homotopy classes, and HUANR is the full subcategory of HUM whose objects are metric spaces 
that have the uniform homotopy type of a uniform ANR. Thus obtained abstract shape category is called the uniform shape category (see [7] for more details).

\section{UNIFORM FIBRATIONS AND UNIFORM SHAPE FIBRATIONS}

Based on the notion of uniform homotopy, there is a corresponding notion of fibration.

A uniformly continuous map $p: E \rightarrow B$ between metric spaces is said to have the uniform homotopy lifting property (UHLP) with respect to a metric space $X$ provided if $h: X \rightarrow E$ and $H: X * I \rightarrow B$ are uniformly continuous maps such that

$$
p \circ h=H(\cdot, 0),
$$

there is a uniformly continuous map $\tilde{H}: X * I \rightarrow E$ such that

$$
\begin{aligned}
& \tilde{H}(\cdot, 0)=h, \text { and } \\
& p \circ \tilde{H}=H .
\end{aligned}
$$

A uniformly continuous map with the UHLP with respect to any metric space is called a uniform fibration. Analogously to Hurewicz fibration, the factorization axiom holds for the uniform fibration.

Proposition 4.1. Every uniformly continuous map $f: X \rightarrow Y$ between metric spaces is the composite of a uniform homotopy equivalence $h: X \rightarrow E$ and a uniform fibration $g: E \rightarrow Y$ for some metric space $E$.

Proof. Let $E_{f}$ be the subspace of $X \times U(I, Y)$ consisting of all pairs $(x, \omega)$ such that $f(x)=\omega(0)$, where $X \times U(I, Y)$ is the uniform product. Define maps $h: X \rightarrow E_{f}$ and $g: E_{f} \rightarrow Y$ by $h(x)=\left(x, e_{f(x)}\right)$ and $g(x, \omega)=$ $\omega(1)$, respectively. Then $h$ is a uniform homotopy equivalence since the map $p: E_{f} \rightarrow X$ defined by $p(x, \omega)=x$ is a uniform homotopy inverse of $h$. To see that the map $g$ is a uniform fibration, consider the commutative diagram

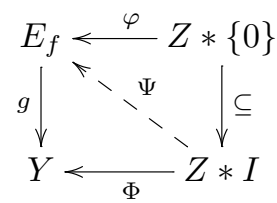

where $\varphi$ and $\Phi$ are uniformly continuous maps. Write $\varphi(z)=\left(\varphi_{1}(z), \varphi_{2}(z)\right) \in$ $X \times U(I, Y)$. Then the map $\Psi$ is defined by $\Psi(z, t)=\left(\Psi_{1}(z, t), \Psi_{2}(z, t)\right) \in$ $X \times U(I, Y)$, where

$$
\begin{aligned}
& \Psi_{1}(z, t)=\varphi_{1}(z), \\
& \Psi_{2}(z, t)= \begin{cases}\varphi_{2}(z)(s(1+t)) & 0 \leq s \leq \frac{1}{1+t}, \\
\Phi(z,(1+t) s-1) & \frac{1}{1+t} \leq s \leq 1 .\end{cases}
\end{aligned}
$$


That $\Psi$ is a uniformly continuous map follows from the bijection $U(X * I, Y) \approx$ $U(X, U(I, Y))$ (see Proposition $3.1(1)$ ).

Similarly to the topological case, one can consider two kinds of homotopy lifting property, which we call the uniform homotopy lifting property and the approximate uniform homotopy lifting property, respectively.

A level morphism $\left(g_{i}\right): \boldsymbol{E} \rightarrow \boldsymbol{B}$ between UANR-towers $\boldsymbol{E}=\left(E_{i}, q_{i, i+1}\right)$ and $\boldsymbol{B}=\left(B_{i}, r_{i, i+1}\right)$ is said to have the uniform homotopy lifting property (UHLP) with respect to a metric space $X$ provided for every $i \in \mathbb{N}$, there exists a $j=j(i) \geq i$ such that whenever $h_{j}: X \rightarrow E_{j}$ and $H_{j}: X * I \rightarrow B_{j}$ are uniformly continuous maps satisfying

$$
g_{j} \circ h_{j}=H_{j}(\cdot, 0),
$$

then there is a uniformly continuous map $\tilde{H}_{i}: X * I \rightarrow E_{i}$ satisfying

$$
\begin{aligned}
& \tilde{H}_{i}(\cdot, 0)=q_{i j} \circ h_{j} \text {, and } \\
& g_{i} \circ \tilde{H}_{i}=r_{i j} \circ H_{j} .
\end{aligned}
$$

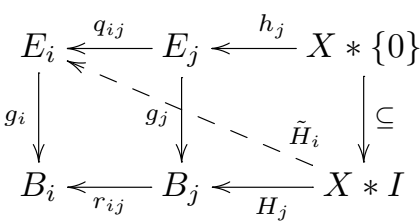

A level morphism $\left(g_{i}\right): \boldsymbol{E} \rightarrow \boldsymbol{B}$ is said to have the approximate uniform homotopy lifting property (AUHLP) with respect to a metric space $X$ provided for every $i \in \mathbb{N}$ and for every $\varepsilon>0$, there exist a $j=j(i) \geq i$ and $\delta>0$ such that whenever $h_{j}: X \rightarrow E_{j}$ and $H_{j}: X * I \rightarrow B_{j}$ are uniformly continuous maps satisfying

$$
d\left(g_{j} \circ h_{j}, H_{j}(\cdot, 0)\right)<\delta,
$$

then there is a uniformly continuous map $\tilde{H}_{i}: X * I \rightarrow E_{i}$ satisfying

$$
\begin{aligned}
& d\left(\tilde{H}_{i}(\cdot, 0), q_{i j} \circ h_{j}\right)<\varepsilon, \text { and } \\
& d\left(g_{i} \circ \tilde{H}_{i}, r_{i j} \circ H_{j}\right)<\varepsilon .
\end{aligned}
$$

Obviously, the UHLP implies the AUHLP. However, the UHLP is not invariant for level morphisms between UANR-towers that induce the same uniformly continuous map, while the AHLP is invariant. More precisely, we have

Proposition 4.2. If $(\boldsymbol{q}, \boldsymbol{r}, \boldsymbol{g})$ and $\left(\boldsymbol{q}^{\prime}, \boldsymbol{r}^{\prime}, \boldsymbol{g}^{\prime}\right)$ are uniform resolutions of a uniformly continuous map $g: E \rightarrow B$ between metric spaces, then whenever $\boldsymbol{g}$ has the AHLP, so does $\boldsymbol{g}^{\prime}$. $[5]$.

Proof. The assertion is proved by an argument similar to Theorem 1 of 
A uniformly continuous map $g: E \rightarrow B$ between metric spaces is a uniform shape fibration provided it admits a uniform resolution $(\boldsymbol{q}, \boldsymbol{r}, \boldsymbol{g})$ such that $\boldsymbol{q}: E \rightarrow \boldsymbol{E}$ and $\boldsymbol{r}: B \rightarrow \boldsymbol{B}$ are UANR-resolutions of $E$ and $B$, respectively, and the level morphism $\boldsymbol{g}: \boldsymbol{E} \rightarrow \boldsymbol{B}$ has the AUHLP with respect to any metric space $X$. Proposition 4.2 guarantees that the property of being a uniform shape fibration does not depend on the choice of the uniform resolution.

A uniform shape fibration is a generalization of a uniform fibration between uniform ANR's. Indeed, if $g: E \rightarrow B$ is a uniformly continuous map between uniform ANR's which has the UHLP, the level morphism $(g):(E) \rightarrow(B)$ between rudimentary systems has the UHLP. A uniformly continuous map between metric spaces that admits a uniform resolution $(\boldsymbol{q}, \boldsymbol{r}, \boldsymbol{g})$ such that the level morphism $\boldsymbol{g}=\left(g_{i}\right)$ consists of the uniform fibrations $g_{i}$ is a uniform shape fibration.

\section{Proof of the MaIn theorem}

Let $f: X \rightarrow Y$ be a uniformly continuous map between metric spaces $X$ and $Y$, and let $(\boldsymbol{p}, \boldsymbol{q}, \boldsymbol{f})$ be the uniform resolution of $f$ defined in Theorem 2.2. Let $E_{f}$ be as in the proof of Proposition 4.1, and for each $\varepsilon>0$, let $E_{f}^{\varepsilon}$ be the subset of $X \times U(I, Y)$ consisting of all pairs $(x, \omega)$ so that $d(f(x), \omega(0))<\varepsilon$, where $X \times U(I, Y)$ is the uniform product.

Let $\left\{\varepsilon_{i}: i \in \mathbb{N}\right\}$ be a sequence of positive numbers satisfying the following properties:

$$
\begin{aligned}
& \varepsilon_{1}<1 / 2 \text {, and } \varepsilon_{i+1}<\min \left\{1 / 2^{i+1}, \varepsilon_{i}\right\} \text { for } i \in \mathbb{N} \text {, and } \\
& \text { any } \varepsilon_{i+1} \text {-near uniformly continuous maps into } Y_{i} \text { are } \\
& \text { uniformly } \varepsilon_{i} \text {-homotopic. }
\end{aligned}
$$

For each $i \in \mathbb{N}$, define uniformly continuous maps $r_{i}: E_{f} \rightarrow E_{f_{i}}^{\varepsilon_{i}}, r_{i, i+1}:$ $E_{f_{i}}^{\varepsilon_{i+1}} \rightarrow E_{f_{i}}^{\varepsilon_{i}}, h_{i}: X_{i} \rightarrow E_{f_{i}}^{\varepsilon_{i}}$, and $g_{i}: E_{f_{i}}^{\varepsilon_{i}} \rightarrow Y_{i}$ as follows:

$$
\begin{aligned}
& r_{i}(x, \omega)=\left(p_{i}(x), q_{i} \circ \omega\right) \text { for each }(x, \omega) \in E_{f}, \\
& r_{i, i+1}(x, \omega)=\left(p_{i, i+1}(x), q_{i, i+1} \circ \omega\right) \text { for each }(x, \omega) \in E_{f_{i}}^{\varepsilon_{i+1}}, \\
& h_{i}(x)=\left(x, e_{f_{i}(x)}\right) \text { for each } x \in X_{i}, \\
& g_{i}(x, \omega)=\omega(1) \text { for each }(x, \omega) \in E_{f_{i}}^{\varepsilon_{i}} .
\end{aligned}
$$

Each $E_{f_{i}}^{\varepsilon_{i}}$ is a uniform ANR since it is a uniform neighborhood of $E_{f}$ in the uniform ANR $X_{i} \times U\left(I, Y_{i}\right)$. To see that $E_{f_{i}}^{\varepsilon_{i}}$ is a uniform neighborhood, let $\delta>0$ be such that $\delta<\varepsilon_{i} / 2$ and such that for any $x, x^{\prime} \in X_{i}$, if $f\left(x, x^{\prime}\right)<\delta$, then $d\left(f_{i}(x), f_{i}\left(x^{\prime}\right)\right)<\varepsilon_{i} / 2$. Then if $\left(x^{\prime}, \omega^{\prime}\right) \in X_{i} \times U\left(I, Y_{i}\right)$ and if $d\left(x, x^{\prime}\right)<\delta$ and $d\left(\omega, \omega^{\prime}\right)<\delta$ for some $(x, \omega) \in E_{f}$, then $d\left(f_{i}\left(x^{\prime}\right), \omega^{\prime}(0)\right) \leq d\left(f_{i}\left(x^{\prime}\right), f_{i}(x)\right)+$ $d\left(f_{i}(x), \omega(0)\right)+d\left(\omega(0), \omega^{\prime}(0)\right)<\varepsilon_{i}$, showing $\left(x^{\prime}, \omega^{\prime}\right) \in E_{f_{i}}^{\varepsilon_{i}}$. 
The following diagram commutes:

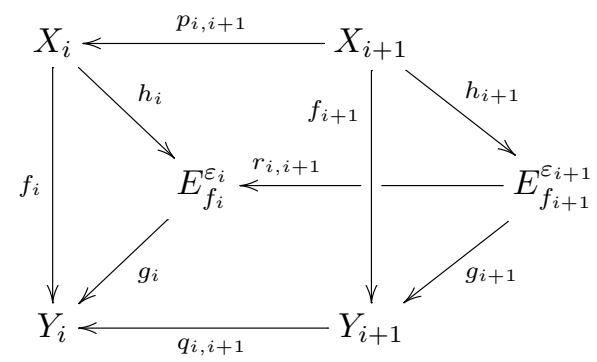

Thus, the UANR-tower $\boldsymbol{E}=\left(E_{f_{i}}^{\varepsilon_{i}}, r_{i, i+1}\right)$ is well-defined, and the following diagram commutes:

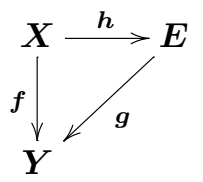

Since $r_{i, i+1} \circ r_{i+1}=r_{i}$, the morphism $\boldsymbol{r}=\left(r_{i}\right): E_{f} \rightarrow \boldsymbol{E}$ is well-defined.

Claim 1. The morphism $\boldsymbol{r}=\left(r_{i}\right): E_{f} \rightarrow \boldsymbol{E}$ is a UANR-resolution.

To show Claim 1, it suffices to show that for each $\varepsilon>0$, there exists $i \in \mathbb{N}$ such that $E_{f_{i}}^{\varepsilon_{i}}$ is contained in the $\varepsilon$-neighborhood of $E_{f}$. Then conditions $\left(\mathrm{R}_{1}\right)$ and $\left(\mathrm{R}_{2}\right)$ easily follow from an argument similar to the proof of Theorem 2.2 (1).

Let $\varepsilon>0$. Let $r: \ell^{\infty}(Y) \rightarrow Y$ be a uniform retraction, and take $\delta>0$ such that

$$
\delta<\varepsilon \text {, and }
$$

$$
\text { for any } y, y^{\prime} \in \ell^{\infty}(Y) \text {, if } d\left(y, y^{\prime}\right)<\delta \text {, then } d\left(r(y), r\left(y^{\prime}\right)\right)<\varepsilon / 4 \text {. }
$$

There exists $i \in \mathbb{N}$ with the following properties (see (5.1)):

$$
\begin{aligned}
& \varepsilon_{i}<\delta / 4, \text { and } \\
& \text { if } d\left(x, x^{\prime}\right)<\varepsilon_{i}, \text { then } d\left(\bar{f}(x), \bar{f}\left(x^{\prime}\right)\right)<\delta / 4 .
\end{aligned}
$$

To see that $E_{f_{i}}^{\varepsilon_{i}}$ is contained in the $\varepsilon$-neighborhood of $E_{f}$, let $(x, \omega) \in E_{f_{i}}^{\varepsilon_{i}}$. We must show that there exists $\left(x^{\prime}, \omega^{\prime}\right) \in E_{f}$ such that $d\left(x, x^{\prime}\right)<\varepsilon$ and $d\left(\omega, \omega^{\prime}\right)<\varepsilon$.

Take $x^{\prime} \in X$ so that $d\left(x, x^{\prime}\right)<\varepsilon_{i}$. Then $d\left(x, x^{\prime}\right)<\varepsilon$ by (5.11) and (5.9).

We define the path $\omega^{\prime}$ in $Y$ as follows. Since $d\left(f_{i}\left(x^{\prime}\right), \omega(0)\right) \leq$ $d\left(f_{i}\left(x^{\prime}\right), f_{i}(x)\right)+d\left(f_{i}(x), \omega(0)\right)<\delta / 4+\varepsilon_{i}<\delta / 2$ by (5.12) and (5.11), then there is a path $\sigma$ from $f_{i}\left(x^{\prime}\right)$ to $\omega(0)$ in $\ell^{\infty}(Y)$ whose image lies in the $\delta / 2$-ball 
centered at $\omega(0)$. Define a path $\omega^{\prime \prime}$ in $\ell^{\infty}(Y)$ by

$$
\omega^{\prime \prime}(t)= \begin{cases}\sigma(n t) & 0 \leq t \leq \frac{1}{n} \\ \omega\left(\frac{n t-1}{n-1}\right) & \frac{1}{n} \leq t \leq 1\end{cases}
$$

where $n$ is a positive integer satisfying the following two conditions:

- $\omega\left(\left[0, \frac{1}{n}\right]\right)$ is contained in the $\delta / 2$-ball centered at $\omega(0)$,

- $\omega\left(\left[\frac{i}{n-1}, \frac{i+1}{n-1}\right]\right)$ is contained in some open $\delta / 2$-ball.

Then

$$
d\left(\omega, \omega^{\prime \prime}\right)<\delta
$$

Indeed, for each $t \in\left[0, \frac{1}{n}\right], d(\sigma(n t), \omega(t))<\delta$ since $\sigma(n t)$ and $\omega(t)$ are points in the open $\delta / 2$-ball centered at $\omega(0)$; for each $t \in\left[\frac{1}{n}, 1\right], d\left(\omega\left(\frac{n t-1}{n-1}\right), \omega(t)\right)<$ $\delta$ since $\left|t-\frac{n t-1}{n-1}\right| \leq \frac{1}{n-1}$. Now define the path $\omega^{\prime}$ in $Y$ by $\omega^{\prime}=r \circ \omega^{\prime \prime}$. Then $\omega^{\prime}(0)=f\left(x^{\prime}\right)$. It remains to show that $d\left(\omega^{\prime}, \omega\right)<\varepsilon$. Indeed, this inequality follows from the inequality $d\left(r \circ \omega, \omega^{\prime}\right)<\varepsilon / 4$ by (5.13) and (5.10), and $d(r \circ \omega, \omega)<\varepsilon / 2$ (for each $t \in I, d\left(\omega(t), y_{t}\right)<\varepsilon_{i}<\delta / 4$ for some $y_{t} \in Y$, so $d(r \circ \omega(t), \omega(t)) \leq d\left(r \circ \omega(t), y_{t}\right)+d\left(\omega(t), y_{t}\right)<\varepsilon / 2$ by $(5.10)$ and $\left.(5.9)\right)$. This proves the claim.

The level morphisms $\boldsymbol{h}=\left(h_{i}\right): \boldsymbol{X} \rightarrow \boldsymbol{E}$ and $\boldsymbol{g}=\left(g_{i}\right): \boldsymbol{E} \rightarrow \boldsymbol{Y}$ induce uniformly continuous maps $h: X \rightarrow E_{f}: x \mapsto\left(x, e_{f(x)}\right)$ and $g: E_{f} \rightarrow Y$ : $(x, \omega) \mapsto \omega(1)$, and diagram (5.8) induces the equality $f=g \circ h$.

Claim 2. $g$ is a uniform shape fibration.

This follows from the fact that each $g_{i}: E_{f_{i}}^{\varepsilon_{i}} \rightarrow Y_{i}$ has the UHLP with respect to any metric space $Z$, which is proved by the same argument as for Proposition 4.1.

Claim 3. $h$ induces a uniform shape equivalence.

It suffices to show that there is a uniformly continuous map $\beta_{i}: E_{f i+1}^{\varepsilon_{i+1}} \rightarrow$ $X_{i}$ that makes the following diagram commute up to uniform homotopy:

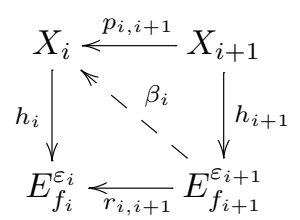

Let $\alpha_{i}: E_{f_{i}}^{\varepsilon_{i}} \rightarrow X_{i}$ be the restriction of the projection of $X_{i} \times U\left(I, Y_{i}\right)$ onto $X_{i}$, and define the map $\beta_{i}: E_{f_{i+1}}^{\varepsilon_{i+1}} \rightarrow X_{i}$ by $\beta_{i}=\alpha_{i} \circ r_{i, i+1}$. Then obviously $\beta_{i} \circ h_{i+1}=p_{i, i+1}$. To see that $h_{i} \circ \beta_{i}$ is uniformly homotopic to $r_{i, i+1}$, note that $h_{i} \circ \beta_{i}(x, \omega)=\left(p_{i, i+1}(x), e_{f_{i} \circ p_{i, i+1}(x)}\right)$ and $r_{i, i+1}(x, \omega)=\left(p_{i, i+1}(x), q_{i, i+1} \circ \omega\right)$ 
for $(x, \omega) \in E_{f_{i+1}}^{\varepsilon_{i+1}}$. Since $d\left(f_{i+1}(x), \omega(0)\right)<\varepsilon_{i+1}$ for each $(x, \omega) \in E_{f_{i+1}}^{\varepsilon_{i+1}}$, there is a uniform $\varepsilon_{i}$-homotopy $K: E_{f_{i+1}}^{\varepsilon_{i+1}} \times I \rightarrow Y_{i}$ such that $K(x, \omega, 0)=$ $p_{i, i+1} \circ f_{i+1}(x)$ and $K(x, \omega, 1)=q_{i, i+1} \circ \omega(0)$ (by (5.2)). Define a uniformly continuous map $H: E_{f_{i}}^{\varepsilon_{i}} * I \rightarrow E_{f_{i}}^{\varepsilon_{i}}$ by

$$
H((x, \omega), t)= \begin{cases}\left(x, e_{K(x, \omega, 2 t)}\right) & 0 \leq t \leq \frac{1}{2}, \\ \left(x, q_{i, i+1} \circ \omega_{2 t-1}\right) & \frac{1}{2} \leq t \leq 1 .\end{cases}
$$

Here, for each $t \in I$, the path $\omega_{t}: I \rightarrow Y_{i}$ is defined by $\omega_{t}(s)=\omega(s t)$ for $s \in I$. Then this gives a uniform homotopy between $h_{i} \circ \beta_{i}$ and $r_{i, i+1}$.

\section{REFERENCES}

[1] D. S. Coram and P. F. Duvall, Jr., Approximate fibrations, Rocky Mountain J. Math. 7 (1977), 275-288.

[2] S. T. Hu, Theory of retracts, Wayne State University Press, Detroit, 1965.

[3] J. R. Isbell, Uniform spaces, AMS, Providence, 1964.

[4] S. Mardešić, Approximate polyhedra, resolutions of maps and shape fibrations, Fund. Math. 114 (1981), 53-78.

[5] S. Mardešić and T. B. Rushing, Shape fibrations. I, General Topology Appl. 9 (1978), 193-215.

[6] S. Mardešić and J. Segal, Shape theory, North Holland Publishing Company, Amsterdam-New York, 1982.

[7] T. Miyata, Uniform shape theory, Glas. Mat. Ser. III 29(49) (1994), 123-168.

\section{T. Miyata}

Department of Mathematics and Informatics

Graduate School of Human Development and Environment

Kobe University

3-11 Tsurukabuto, Nada-Ku, Kobe

657-8501 Japan

E-mail: tmiyata@kobe-u.ac.jp

Received: 6.5.2014.

Revised: 29.5.2014. 